\title{
Association of leptin with cardiometabolic factors in schoolchildren and adolescents with congenital adrenal hyperplasia
}

\author{
Jessie Nallely Zurita-Cruz', Miguel Ángel Villasís-Keever², Leticia Damasio-Santana, \\ Leticia Manuel-Apolinar ${ }^{3}$, Rosalba Ferrusca-Ceja ${ }^{4}$, Elisa Nishimura-Meguro ${ }^{4}$, \\ Aleida de J. Rivera-Hernández ${ }^{4}$ and Eulalia Garrido-Magaña ${ }^{4}$ \\ ${ }^{1}$ Unit of Research in Medical Nutrition; '2Unit of Medical Research in Clinical Epidemiology; ${ }^{3}$ Unit of Medical Research in Endocrine Diseases, \\ Diabetes and Metabolism; ' ${ }^{P}$ Pediatrics Hospital, Department of Pediatric Endocrinology, Instituto Mexicano del Seguro Social, Centro Médico Nacional \\ Siglo XXI, Ciudad de México, Mexico
}

\begin{abstract}
Introduction: In congenital adrenal hyperplasia (CAH), obesity, hyperinsulinemia and leptin levels are increased. Objective: To identify the frequency of cardiometabolic risk factors (CRF) in children and adolescents with CAH and to explore the relationship with leptin levels. Method: Cross-sectional study of 40 patients who underwent anthropometric measurements and had fasting glucose, insulin, triglycerides, 17-hidroxyprogesterone, leptin, HDL and LDL-cholesterol assessed. The patients were classified according to the number of CRFs, and leptin levels were analyzed with the Kruskal-Wallis test. Pearson's correlation was applied between leptin, body mass index (BMI) z-score and body fat percentage. Results: Fifty percent of the patients had obesity and overweight, 59\% had hypertriglyceridemia, 40\%, hypoalphalipoproteinemia, 27.5\%, high LDL-cholesterol and $22.5 \%$ insulin resistance. There was positive correlation between leptin and body fat percentage $(r=0.64), B M I z$-score $(r=0.55)$ and the number of CRFs $(r=0.65)$. In the obesity-adjusted multivariate analysis, leptin levels were associated with the number of CRFs. Conclusion: $\mathrm{CAH}$ had a high frequency of CRFs and leptin appeared to be associated with a more adverse cardiometabolic profile in subjects with obesity and overweight.
\end{abstract}

KEY WORDS: Congenital adrenal hyperplasia. Leptin. Pediatrics.

\section{Introduction}

Congenital adrenal hyperplasia $(\mathrm{CAH})$ is a recessive autosomal disorder characterized by increased androgen production and cortisol deficiency. ${ }^{1}$ The goal of treatment is to provide adequate cortisol replacement in order to prevent adrenal crises and to suppress androgen excessive production. ${ }^{2}$ However, in order for this effect to be achieved, supraphysiological cortisol doses have to be administered, by means of which the line between endogenous hyperandrogenism suppression and Cushing syndrome iatrogenic induction is very thin. ${ }^{3}$
Studies in adults with CAH have demonstrated high prevalence of obesity, ${ }^{4}$ hypercholesterolemia, insulin resistance $(\mathrm{IR})^{5}$ and arterial hypertension; ${ }^{6}$ cortisol supraphysiological doses can be a possible triggering factor in the development of cardiometabolic risk factors (CRF). ${ }^{7}$

On the other hand, Völkl et al. described 89 German children and adolescents with $\mathrm{CAH}$ in whom obesity was identified in $16.8 \%$, which is a higher proportion in comparison with that reported in children and adolescents of the German general population (2.27\%). ${ }^{8}$ In a study that included 22 patients with ages ranging from 7 to 22 years, higher values were observed in the BMI Z-score (BMIz) than in healthy population. ${ }^{9}$
Correspondence:

Jessie Nallely Zurita-Cruz

E-mail: zuritajn@ hotmail.com
Date of reception: 06-05-2017

Date of acceptance: 18-05-2017

DOI://dx.doi.org/10.24875/GMM.M18000131
Gac Med Mex. 2018;154:157-162

Contents available at PubMed www.gacetamedicademexico.com 
Leptin is a peptide hormone ${ }^{10}$ that is produced by adipocytes, regulated by hypothalamic neuropeptides $^{11}$ and that is implicated in energy metabolism, thermogenesis and weight gain. ${ }^{12}$ In human beings, leptin has been associated with obesity and is considered to be a risk factor for metabolic syndrome. ${ }^{13}$ Leptin plays a permissive role in pubertal development. Some studies associate puberty onset with an increase of leptin levels. ${ }^{14}$ These actions can explain why leptin levels are different according to the age, gender, body fat and puberty stage. Both obesity and puberty can be associated with leptin increase. $^{15}$

Particularly in patients with $\mathrm{CAH}$, high androgen serum concentrations alter leptin metabolism, which causes hyperleptinemia; on the other hand, $\beta$-adrenergic receptors, which regulate lipid metabolism and thermogenesis, are deficient in patients with $\mathrm{CAH} .{ }^{16}$ This induces an adverse environment, which elicits an increase in the development of CRFs.

Adults and adolescents with $\mathrm{CAH}$ have been described to show a higher prevalence of CRFs, with leptin levels being associated with some of them. Whether CRFs and leptin are elevated since the pre-pubertal stage has thus far not been studied, and neither has the relationship between the number of CRFs and leptin levels.

The purpose of this study was to identify the prevalence of CRFs in schoolchildren and adolescents with $\mathrm{CAH}$ and their relationship with leptin levels.

\section{Method}

This was a cross-sectional study of Mexican children and adolescents that was carried out between December 2014 and July 2015. Patients with ages ranging from 6 to 16 years, who underwent follow-up for at least 12 months by the pediatric endocrinology department and who received systemic steroid-based hormone replacement were included. Forty-six patients who met the inclusion criteria were selected; 6 were excluded: 2 had incomplete exams, and in 4, the parents denied permission for their children to participate in the study. The research protocol was approved by the hospital research committee with registry number R-20143603-32. Parents signed the informed consent letter and patients older than 8 years signed the letter of assent, according to recommendations of the Declaration of Helsinki.

\section{Clinical and anthropometric assessment}

Recorded somatometric variables included weight, height, waist circumference and body fat percentage (BF\%) measured by the bioimpedance method (Tanita $\mathrm{BC}-568)$; these measurements were performed by a certified nutritionist. Pubertal status was identified by physical examination according to the Tanner scale. Blood pressure was the average of 3 measurements taken with a sphygmomanometer on three separate occasions. IR was estimated using the homeostasis model (HOMA): $:^{17}$

$I R=$ insulin (microunits per milliliter) $x$

glucose (millimoles per liter/22.5)

Analyzed CRFs were obesity (BMI at $\geq 95^{\text {th }}$ percentile), systemic arterial hypertension (systolic pressure at $\geq 90^{\text {th }}$ percentile or diastolic pressure at $\geq 90^{\text {th }}$ percentile-for-age), ${ }^{18}$ fasting glucose alteration (plasma glucose $\geq 10 \mathrm{mg} / \mathrm{dL}$ ), ${ }^{19}$ hypertriglyceridemia (plasma triglycerides $[\mathrm{TGL}]$ at $\geq 90^{\text {th }}$ percentile according to age and gender), hypoalphalipoproteinemia (high-density lipoprotein cholesterol [HDL-C] at $<10^{\text {th }}$ percentile-for-age and gender), ${ }^{20}$ low-density lipoprotein cholesterol (LDL-C) > $100 \mathrm{mg} / \mathrm{dL}$ and IR (HOMA score > 3.4)..$^{21}$ Body fat was considered to be abnormal according to gender: in females, when it was $>30 \%$, and in males, $>20 \%{ }^{22,23}$

The glucocorticoid dose was expressed as the accumulated dose per body surface area (BSA) per day (milligrams per square meter $\left[\mathrm{mg} / \mathrm{m}^{2} \mathrm{BSA}\right]$ ), taking the dose average of the last 6 months prior to the study. All glucocorticoid doses were converted to hydrocortisone $(\mathrm{HC})$ doses: $1 \mathrm{mg}$ prednisone $=4 \mathrm{mg} \mathrm{HC}$ and $1 \mathrm{mg}$ prednisolone $=5 \mathrm{mg} \mathrm{HC}$. CAH was regarded as being adequately controlled when 17-hydroxiprogesterone (17OHP) was $<10 \mathrm{ng} / \mathrm{mL}$.

\section{Biochemical assessment}

Sera were obtained from blood samples taken by means of venipuncture after a 12-h fast. Glucose, TGL, LDL-C and HDL-C were determined using colorimetric enzymatic methods (Bayer diagnostics, Puteaux, France). Insulin was measured by chemoluminescence (Roche-Hitachi modular P and D), $170 \mathrm{HP}$ and leptin (Human Leptin Duo Set, DY 398 ${ }^{\circledR}$ ) were measured with enzyme-linked immunosorbent assay (ELISA) (R \& D Systems, Minneapolis, MN, USA). All ELISA analyses were determined using Finstruments Multiskan EX, MTX ${ }^{\bullet}$ (Lab Systems), in duplicate, as 
recommended by the manufacturer. Intra- and inter-assay variation coefficient was $<7 \%$ for all measurements. A standardization curve was also included for each assay.

\section{Statistical analysis}

Data were analyzed using the SPSS version 17.0 statistical program. Since no normal distribution was found for quantitative variables, the mean and minimum and maximum values were calculated.

Patients were classified according to the number of CRFs, and leptin levels were compared with Kruskal-Wallis test. The correlation between leptin and $\mathrm{BMI}$ and BF\% was determined with Pearson's coefficient. In order to identify variables associated with predicted CRF number, different linear regression models were constructed, where leptin, 17OHP, HC dose, absence or presence of puberty, BMIz and BF\% were included.

\section{Results}

Forty patients ( 26 females), with ages ranging from 6 to 16 years (median of 11.9 years) were analyzed. The bone age/chronologic age ratio was higher than one in most cases, owing to an advanced bone age in these patients. CAH most common variant was the salt-losing type, followed by simple virilization. All patients received replacement treatment with glucocorticoids, with a median $\mathrm{HC}$ dose of $15.7 \mathrm{mg} / \mathrm{m}^{2} \mathrm{BSA}$ (Table 1).

Regarding CRFs, 14 (35\%) and 8 (20\%) subjects had obesity and overweight, respectively; hypertriglyceridemia was identified in 20 (59\%), and 16 (40 $\%)$ had hypoalphalipoproteinemia, 11 (27.5\%), LDL-C increase, 9 (22.5\%), IR, 3 (7.5\%), systemic arterial hypertension and one had altered fasting glucose. Of the 40 subjects, $33(77.5 \%)$ had on or more CRFs; specifically, $25 \%$ had one factor, $20 \%$, two, $17.5 \%$, three, $7.5 \%$, four, and $7.5 \%$ had five. BF\% was found to be above normal in $65 \%(n=26)$ of patients (Table 2).

In total, only $25 \%$ of patients $(n=10)$ had no CRFs, $17.5 \%(n=7)$ had one factor and $57.5 \%(n=23)$ had more than two. The number of CRFs was compared between patients with and without obesity: $92.8 \%$ $(n=13)$ of patients with obesity had two or more CRFs; of the patients without obesity, only $38.46 \%$ $(n=10)$ had two or more, with statistical significance $(p=0.013)$.
Table 1. General data of 40 pediatric patients with congenital adrenal hyperplasia

\begin{tabular}{|c|c|c|}
\hline & $\mathrm{n}$ & $\%$ \\
\hline \multicolumn{3}{|l|}{ Gender } \\
\hline Female & 26 & $65 \div$ \\
\hline Male & 14 & $35 \geq$ \\
\hline \multicolumn{3}{|l|}{ CAH classification } \\
\hline Salt-losing & 30 & 75 \\
\hline Simple virilization & 2 & 50 \\
\hline Late & 8 & 20 \\
\hline \multicolumn{3}{|l|}{ Glucocorticoid therapy } \\
\hline Dexamethasone & 7 & 17.5 \\
\hline Prednisolone & 9 & $22.5^{\frac{n}{2}}$ \\
\hline Prednisone & 23 & $56.5 \frac{2}{3}$ \\
\hline Hydrocortisone & 1 & 2.5 \\
\hline $\mathrm{CAH}$ adequate control & 16 & 40 \\
\hline \multicolumn{3}{|l|}{ Tanner stage } \\
\hline | & 9 & $25 \frac{}{5}$ \\
\hline$\|$ & 8 & 22.2 \\
\hline III & 4 & $11.1 \frac{}{2}$ \\
\hline IV & 10 & 27.8 \\
\hline \multirow[t]{2}{*}{ V } & 5 & 13.9 \\
\hline & Median & Minimum-maximun \\
\hline Chronologic age (years) & 12 & $6-16 \stackrel{\circ}{=}$ \\
\hline Bone age (years) & 11 & $6-16$ \\
\hline 17-hydroxiprogesterone (ng/mL) & 11.11 & $0.20-444.7$ \\
\hline Glucocorticoid dose ( $\left.\mathrm{mg} / \mathrm{m}^{2} \mathrm{BSA}\right)$ & 15.7 & $4.9-33.1$ \\
\hline
\end{tabular}

$\mathrm{CAH}$, congenital adrenal hyperplasia; $\mathrm{m}^{2} \mathrm{BSA}$, square meter of body surface area.

To identify if patients had CRFs since pre-pubertal stage and their prevalence, a sub-analysis was carried out of 14 subjects with ages ranging from 6 to 9 years, where 10 (71.4\%) had overweight and obesity ( $28.5 \%$ overweight and $42.8 \%$ obesity), 7 (50\%) had hypertriglyceridemia, 6 (42.8\%) had hypoalphalipoproteinemia, $3(21.4 \%)$ had increased LDL-C and $3(21.4 \%)$ had IR; 12 (85.7 \%) had at least one CRF.

There was positive correlation between leptin values, BF\% and BMIz ( $r=0.55)$ (Fig. 1). Leptin levels were also compared and correlated with the number of CRFs, where the higher the number of factors, the higher the leptin level (Fig. 2).

A linear regression model was constructed to identify patient characteristics that might be related to the number of CRFs; leptin, BMIz, BF\%, $170 \mathrm{HP}$ and HC dose were included. The model showed that leptin and 17OHP were significantly associated with the number of CRFs. Subsequently, a model adjusted for puberty status and presence of overweight and obesity was constructed, where leptin levels were independently related to the number of CRFs (Table 3 ). 

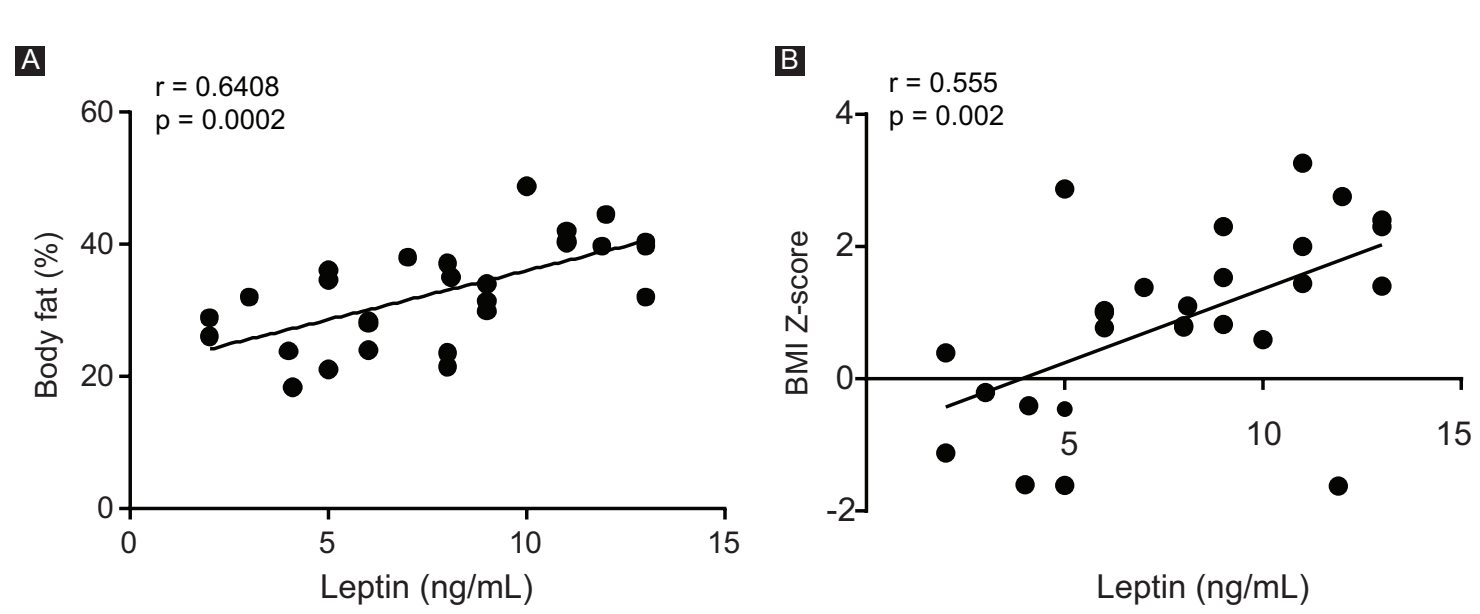

Figure 1. Correlation between leptin levels, body fat percentage $(A)$ and BMI Z-score $(B)$ in patients with congenital adrenal hyperplasia.

Table 2. Somatometric data and biochemical parameters in 40 patients with congenital adrenal hyperplasia

\begin{tabular}{lcc}
\hline & Median & Maximum-minimum \\
\hline BMI Z-score & 0.81 & $-1.62-3.26$ \\
BMlz percentile & 82 & $5-99$ \\
Waist circumference $(\mathrm{cm})$ & 68 & $49-108$ \\
Waist circumference percentile & 50 & $10-90$ \\
Body fat percentage & 30.75 & $18.3-48.8$ \\
Female & 31.7 & $21.5-48.8$ \\
Male & 26 & $18.3-45$ \\
Fasting glucose $(\mathrm{mg} / \mathrm{dL})$ & 85.15 & $66.6-109$ \\
LDL-C (mg/dL) & 82.7 & $11-143$ \\
HDL-C (mg/dL) & 53.2 & $26-77$ \\
Triglycerides $(\mathrm{mg} / \mathrm{dL})$ & 94 & $32-204$ \\
Systolic pressure $(\mathrm{mmHg})$ & 100 & $70-135$ \\
Systolic pressure percentile & 67 & $50-80$ \\
Diastolic pressure $(\mathrm{mmHg})$ & 60 & $50-70$ \\
Diastolic pressure percentile & 50 & $50-90$ \\
Fasting insulin $(\mu \mathrm{IU} / \mathrm{mL})$ & 9.85 & $1.02-37.2$ \\
HOMA & 2.01 & $0.21-9.09$ \\
\hline
\end{tabular}

BMI, body mass index; BMIz, body mass index Z-score; LDL-C, low-density lipoprotein cholesterol; HDL-C, high-density lipoprotein cholesterol.

\section{Discussion}

In this research, patients with CAH had a high prevalence of CRFs, both at the pre-pubertal and pubertal stage. In addition, leptin levels were established to increase according to the number of CRFs. Particularly, in patients with overweight and obesity, leptin levels were associated with an adverse cardiometabolic profile.

\section{Comparison with other studies}

In this study, included patients showed a high prevalence of overweight and obesity (55\%) in comparison with that what is expected for normal pediatric population in Mexico (34.4\%). ${ }^{24}$ CRF prevalence was compared with that reported by Arlt et al., ${ }^{6}$ who describe adult subjects, out of which those with $\mathrm{CAH}$ had a higher prevalence of hypoalphalipoproteinemia (40 versus $14 \%$ ), lower prevalence of LDL-C increase (27.5 versus $39 \%$ ) and similar prevalence of IR (22.5 versus $29 \%$ ), which draws the attention because high prevalence of IR and presence of dyslipidemia are observed since early pediatric stages.

The pathophysiology of CRF development in patients with $\mathrm{CAH}$ is multiple, with supraphysiological steroid doses and hyperandrogenemia standing out, both chronically. The age at which patients develop the incipient clinical and biochemical changes that determine the presence of these cardiovascular and metabolic factors is not known. However, the results suggest that since 6 years of age there are already manifestations.

Puberty is a confounding variable with regard to CRFs. ${ }^{25,26}$ IR increase is related to puberty onset owing to a physiological increase of counter-regulatory hormones that are necessary for maturation and growth. ${ }^{27}$ For this reason, a sub-analysis of CRFs was performed in pre-pubertal patients, where they were observed to have a high prevalence of CRFs; howev$\mathrm{er}$, in the multivariate analysis, puberty was not shown to be associated with an increase in CRFs; therefore, puberty was not a variable related to CRFs.

Obesity-associated mechanisms (BF\% increase, ${ }^{16}$ IR and leptin elevation) are diverse, particularly in this 

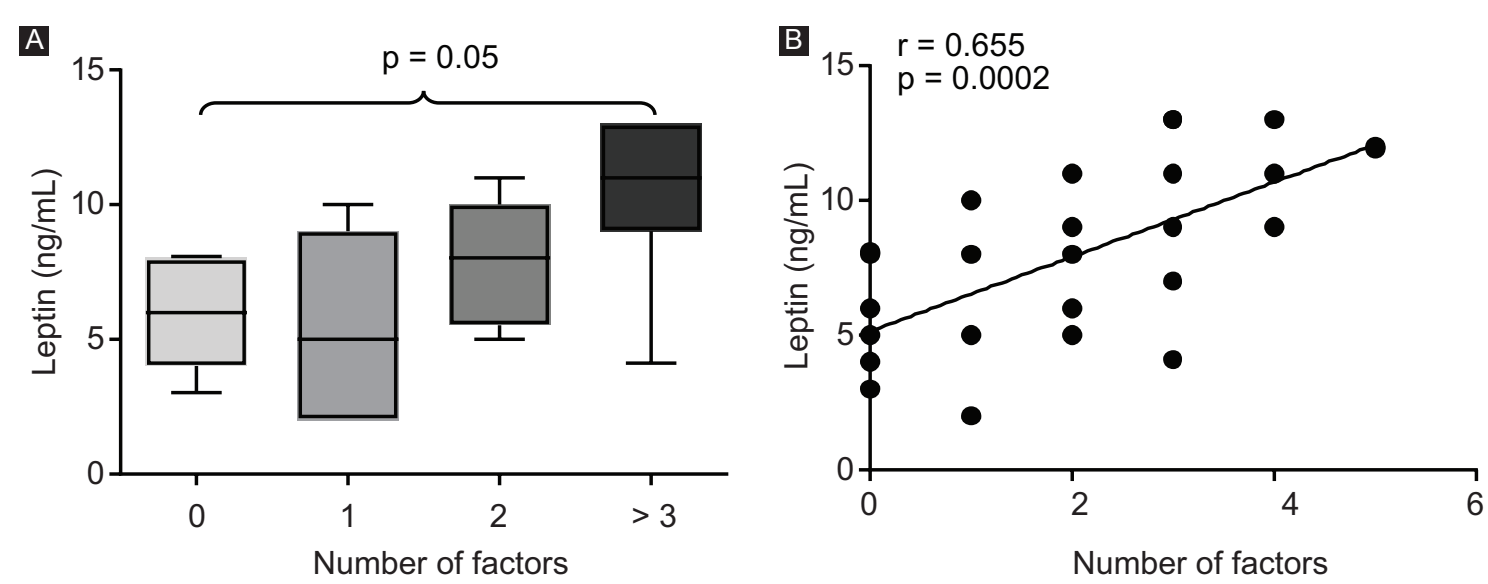

Figure 2. Comparison $(A)$ and correlation (B) of leptin levels and cardiometabolic risk factors.

Table 3. Linear regression analysis to predict the number of cardiometabolic risk factors

\begin{tabular}{|c|c|c|c|c|}
\hline & \multicolumn{2}{|c|}{ Initial model } & \multicolumn{2}{|c|}{ Adjusted model } \\
\hline & $\beta^{*}$ & $95 \% \mathrm{Cl}$ & $\beta^{\star *}$ & $95 \% \mathrm{Cl}$ \\
\hline Leptin serum concentrations (ng/mL) & $0.269 \infty$ & $0.097-0.441$ & $0.322 \infty$ & $0.057-0.606$ \\
\hline 17-hydroxiprogesterone (ng/mL) & $0.005 \infty$ & $0.0001-0.010$ & 0.004 & $-0.003-0.012$ \\
\hline Glucocorticoid dose (mg/m² BSA) & -0.074 & $-0.166-0.011$ & 0.099 & $0.099-0.09$ \\
\hline BMI Z-score & -0.037 & $-0.451-0.376$ & -0.219 & $-0.861-0.423$ \\
\hline Body fat (\%) & -0.003 & $-1.96-3.68$ & -0.014 & $-0.19-0.16$ \\
\hline $\begin{array}{l}\infty p<0.5 \\
* \text { Linear regression coefficient. } \\
*{ }^{*} \text { Multiple linear regression coefficient adjusted for } c \\
\text { BMI, body mass index; } \mathrm{m}^{2} \mathrm{BSA} \text {, square meter of bo }\end{array}$ & and puber & & & 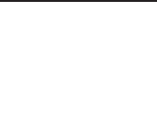 \\
\hline
\end{tabular}

group of subjects. Excess of androgens, such as dehydroepiandosterone and testosterone, can interfere in leptin metabolism via its signaling, which causes a decrease in leptin $\mathrm{OB}$ receptor, thus originating peripheral resistance and, consequently, hyperinsulinism, insulin resistance and obesity, which in turn alters steroidogenesis and increases the production of adrenal ${ }^{28-30}$ and ovarian ${ }^{31}$ androgens.

Obesity and $\mathrm{CAH}$ are associated with high levels of leptin and deficiency of $\mathrm{a-adrenergic} \mathrm{receptors,} \mathrm{which}$ modulate lipid metabolism and thermogenesis, which in turn stimulates the development of CRFs. ${ }^{32,33}$ This induces a pro-inflammatory environment, increased risk for metabolic syndrome and polycystic ovary, as well as a decrease in glucocorticoid treatment efficacy. This explains the high prevalence of CRFs identified in the studied patients.

According to observations, patients with $\mathrm{CAH}$ require early initiation of dietary-environmental and pharmacological intervention since school-age. For example, pharmacological treatment might focus on improving IR by means of sensitizers, such as metformin, ${ }^{34}$ since in these patients there is no way to discontinue the treatment with glucocorticoids or to modify androgen concentration fluctuations, in spite of being controlled.

In this group of patients, which showed a high prevalence of obesity, overweight (55\%) and elevated $\mathrm{BF} \%(65 \%)$, these variables were observed to be confounding with regard to the number of CRFs and leptin levels. Although leptin has been described to be directly related to $\mathrm{BF} \%$ and $\mathrm{BMI},{ }^{31}$ patients with $\mathrm{CAH}$ comprise a particular group of subjects in whom hyperandrogenemia ${ }^{28-32}$ alters the metabolism, including that of leptin, which favors cardiometabolic alterations. This is explained because leptin was an independent variable in the number of CRFs in patients with $\mathrm{CAH}$ and obesity. ${ }^{28}$

\section{Study limitations}

Sample size was small and, therefore, the results should be interpreted as a trend rather than conclusions. Furthermore, since this is a cross-sectional 
study, carrying out a longitudinal analysis would be advisable in order for the age at which CRFs start appearing to be identified. Adipocytokines, in addition to leptin and other anthropometric markers, should be studied in future investigations.

In conclusion, it can be asserted that patients with $\mathrm{CAH}$ have a high prevalence of CRFs and that leptin levels are independently associated with a more adverse cardiometabolic profile in patients with obesity.

\section{References}

1. Speiser PW, White PC. Congenital adrenal hyperplasia. N Engl J Med. 2003;349:776-788.

2. Forest J, Maguelone G. Recent advances in the diagnosis and management of congenital adrenal hyperplasia due to 21-hydroxylase deficiency. Hum Reprod Update 2004:10:469-485.

3. Speiser L, Azziz R, Baskin L, Ghizzoni L, Hensle T, Merke D, et al. Congenital adrenal hyperplasia due to steroid 21-hydroxylase deficiency: an endocrine society clinical practice guideline. J Clin Endocrinol Metab. 2010;95:4133-4160.

4. Kim J, Choi J, Kang E, Kim Y, Lee B, Yoo H. Long-term consequences of congenital adrenal hyperplasia due to classic 21-hydroxylase deficiency in adolescents and adults. Exp Clin Endocrinol Diabetes. 2017:125:196-201

5. Sartorato P, Zulian E, Benedini S, Mariniello B, Schiavi F, Bilora F, et al. Cardiovascular risk factors and ultrasound evaluation of intima-media thickness at common carotids, carotid bulbs, and femoral and abdomina aorta arteries in patients with classic congenital adrenal hyperplasia due to 21-hydroxylase deficiency. J Clin Endocrinol Metab. 2007;92:1015-1018.

6. Arlt W, Willis D, Wild S, Krone N, Doherty E, Hahner S, et al. Health status of adults with congenital adrenal hyperplasia: a cohort study of 203 patients. J Clin Endocrinol Metab. 2010;95:5110-5121.

7. Falhammar H, Frisén L, Hirschberg A, Norrby C, Almqvist C, Nordenskjöld $A$, et al. Increased cardiovascular and metabolic morbidity in patients with 21-hydroxylase deficiency: a Swedish population-based national cohort study. J Clin Endocrinol Metab. 2015;100:3520-3528.

8. Völkl T, Simm D, Beier C, Dörr H. Obesity among children and adolescents with classic congenital adrenal hyperplasia due to 21-hydroxylase deficiency. Pediatrics. 2006;117:e98-e105.

9. Cornean R, Hindmarsh P, Brook C. Obesity in 21-hydroxylase deficient patients. Arch Dis Child. 1998;78:261-263.

10. Baptista C. Leptina. Acta Pediatr Port. 2002;15:281-285.

11. Moran O, Phillip M. Leptin: obesity, diabetes and other peripheral effects-a review. Pediatr Diabetes. 2003:4:101-109.

12. Steinberger J, Steffen L, Jacobs D, Moran A, Hong C, Sinaiko A. Relation of leptin to insulin resistance syndrome in children. Obesity Res. 2003;11:1124-1130

13. Pilcová R, Sulcová J, Hill M, Bláha $P$, Lisá L. Leptin levels in obese children: effects of gender, weight reduction and androgens. Physiol Res. 2003;52:53-60.

14. Sanchez-Garrido M, Tena-Sempere M. Metabolic control of puberty: roles of leptin and kisspeptins. Horm Behav. 2013;64:187-194.

15. Garcia-Mayor R, Andrade M, Rios M, Lage M, Dieguez C, Casanueva F. Serum leptin levels in normal children: relationship to age, gender, body mass index, pituitary-gonadal hormones, and pubertal stage. J Clin Endocrinol Metab. 1997;82:2849-2855.

16. Charmandari E, Weise M, Bornstein S, Eisenhofer G, Keil M, Chrousos G et al.Children with classic congenital adrenal hyperplasia have elevated serum leptin concentrations and insulin resistance: potential clinical implications. J Clin Endocrinol Metab 2002;87:2114-2120.

17. Radziuk J. Insulin sensivity and its measurement: structural commonalities among the methodos. J Clin Endocrinol Metab 2000;85:4426-4433.

18. National High Blood Pressure Education Program Working Group on Hypertension Control in Children and Adolescents. Update on the 1987 Task Force Report on High Blood Pressure in Children and Adolescents: a working group report from the National High Blood Pressure Education Program. Pediatrics. 1996;98:649-658.

19. Zimmet P, Alberti G, Kaufman F, Tajima N, Silink M, Arslanian S, et al. The metabolic syndrome in children and adolescents. Lancet 2007;369:2059-2061.

20. de Ferranti S, Gauvreau K, Ludwig D, Neufeld E, Newburger J, Rifai N. Prevalence of the metabolic syndrome in American adolescents: findings from the Third National Health and Nutrition Examination Survey. Circulation. 2004;110:2494-2497.

21. García-Cuartero B, García-Lacalle C, Jiménez-Lobo C, González-Vergaz A, Calvo-Rey C, Alcázar-Villar M, et al. Indice HOMA y QUICKI, insulina y péptido $C$ en niños sanos. Puntos de corte de riesgo cardiovascular. An Pediatr. 2007:66:481-490.

22. Okasora K, Takaya R, Tokuda M, Fukunaga $Y$, Oguni T, Tanaka H, et al. Comparison of bioelectrical impedance analysis and dual energy X-ray absorptiometry for assessment of body composition in children. Pediatr Int. 1999;41(2):121-125

23. Taylor R, Jones I, Williams S, Goulding A. Body fat percentages measured by dual-energy X-ray absorptiometry corresponding to recently recommended body mass index cutoffs for overweight and obesity in children and adolescents aged 3-18 y. Am J Clin Nutr. 2002; 76(6):1416-1421.

24. Gutiérrez JP, Rivera-Dommarco J, Shamah-Levy T, Villalpando-Hernández S, Franco A, Cuevas-Nasu L, et al. Encuesta Nacional de Salud y Nutrición. México: Instituto Nacional de Salud Pública; 2012. p. 149-152. Disponible en: http://ensanut.insp.mx/informes/ENSANUT2012ResultadosNacionales.pdf

25. Caprio S, Tamborlane W. Effect of puberty on insulin action and secretion. Semin Reprod Endocrinol. 1994;12:90-96.

26. Travers S, Jeffers B, Bloch $C$, Hill J, Eckel R. Gender and tanner stage differences in body composition and insulin sensitivity in early pubertal children. J Clin Endocrinol Metab. 1995;80:172-178.

27. Moran A, Jacobs D, Jr, Steinberger J, Hong C, Prineas R, Luepker R, et al. Insulin resistance during puberty: results from clamp studies in 357 children. Diabetes. 1999;48:2039-2044.

28. L'Allemand D, Penhoat A, Lebrethon M, Ardevol R, Baehr V, Oelkers W, et al. Insulin-like growth factors enhance steroidogenic enzyme and corticotropin receptor messenger ribonucleic acid levels and corticotropin steroidogenic responsiveness in cultured human adrenocortical cells. J Clin Endocrinol Metab. 1996;81:3892-3897.

29. Biason-Lauber A, Zachmann M, Schoenle E. Effect of leptin on CYP17 enzymatic activities in human adrenal cells: new insight in the onset of adrenarche. Endocrinology. 2000;141:1446-1454.

30. Völkl T, Simm D, Körner A, Rascher W, Kiess W, Kratzsch J, et al. Does an altered leptin axis play a role in obesity among children and adolescents with classical congenital adrenal hyperplasia due to 21-hydroxylase deficiency? Eur J Endocrinol. 2009;160:239-247.

31. Hsueh W, Lyon C, Quinones M. Insulin resistance and the endothelium. Am J Med. 2004:117:109-117

32. Merke D, Chrousos G, Eisenhofer G, Weise M, Keil M, Rogol A, et al. Adrenomedullary dysplasia and hypofunction in patients with classic 21-hydroxylase deficiency. N Engl J Med. 2000;343:1362-1368.

33. Gonzaga N, Medeiros C, de Carvalho D, Alves J. Leptin and cardiometabolic risk factors in obese children and adolescents. J Paediatr Child Health. 2014:50(9):707-712.

34. Ibáñez L, Ong K, López-Bermejo A, Dunger D, de Zegher F. Hyperinsulinaemic androgen excess in adolescent girls. Nat Rev Endocrinol. 2014;10(8):499-508 